\title{
Quarante ans d'analyse du travail et de l'emploi : points de vue de quatre économistes
}

Forty Years of Labour Studies: the Perspective of Four Economists

Philippe Askenazy, Luc Behaghel, Morgane Laouenan et Dominique Meurs

\section{OpenEdition}

\section{Journals}

Édition électronique

URL : http://journals.openedition.org/travailemploi/9068

DOI : $10.4000 /$ travailemploi.9068

ISSN : 1775-416X

Éditeur

DARES - Ministère du Travail

Édition imprimée

Date de publication : 2 octobre 2019

Pagination : 69-94

ISSN : 0224-4365

Référence électronique

Philippe Askenazy, Luc Behaghel, Morgane Laouenan et Dominique Meurs, «Quarante ans d'analyse du travail et de l'emploi : points de vue de quatre économistes », Travail et Emploi [En ligne], 158 | 2019, mis en ligne le 01 novembre 2019, consulté le 08 décembre 2020. URL : http://

journals.openedition.org/travailemploi/9068; DOI : https://doi.org/10.4000/travailemploi.9068 


\title{
Quarante ans d'analyse du travail et de l'emploi : points de vue de quatre économistes
}

\author{
Philippe Askenazy, Luc Behaghel, \\ Morgane Laouenan ${ }^{* * *}$, Dominique Meurs ${ }^{* * * *}$
}

\begin{abstract}
Nous mettons en parallèle les mutations du marché du travail en France au cours des quarante dernières années et les évolutions des regards que portent sur lui la statistique publique et les économistes du travail. Trait saillant, l'économie du travail a pris un tournant empirique en mobilisant les nouvelles méthodes micro-économétriques d'analyse des politiques publiques développées à partir des années 1990. Ce tournant n'a pas suffi pour progresser de façon décisive dans la lutte contre le chômage ou les inégalités sur le marché du travail ; on peut s'interroger en retour sur les difficultés de la discipline et de ses outils statistiques à appréhender un contexte mouvant, mais aussi peut-être sur le dialogue encore insuffisant avec les décideurs publics.
\end{abstract}

\begin{abstract}
Comment a évolué le marché du travail en France depuis quarante ans, et comment a évolué la connaissance que nous en avons ? Pour mettre en perspective ces changements, tant dans les faits que dans notre regard, un exercice amusant serait d'endosser les habits du Persan à Paris, et de décrire avec les lunettes des années 1970 le champ de l'économie du travail aujourd'hui en France : que retiendraient Gary Becker et Milton Friedman, deux des penseurs de cette époque qui ont contribué à modeler notre vision actuelle, s'ils venaient à passer un congé sabbatique en France en 2020 ? Plus modestement, cet article présente les points de vue de quatre économistes sur le travail et l'emploi en France depuis quarante ans. Plutôt que de s'opposer, les points de vue reflétés ici convergent pour souligner que ces décennies sont traversées par trois lames de fond : celle d'un marché du travail en mutations accélérées, suivies vaille que vaille par l'appareil statistique ; celle d'inflexions fortes des politiques de l'emploi et de leurs évaluations ; enfin, un renouvellement profond de l'analyse des discriminations.
\end{abstract}

\footnotetext{
* CNRS-ENS, Centre Maurice-Halbwachs ; philippe.askenazy@ens.fr.

** PSE-École d'économie de Paris, Inra ; luc.behaghel@ens.fr.

*** Centre d'économie de la Sorbonne, CNRS, Sciences Po Liepp ; morgane.laouenan@univ-paris1.fr.

**** Université Paris Nanterre et chaire Travail PSE ; dominique.meurs@ parisnanterre.fr.
} 


\section{Les grandes mutations du marché du travail et de son observation}

Le marché du travail en France a connu de profonds bouleversements en quatre décennies que ce soit du côté de l'offre, de celui de la demande, ou de celui de son fonctionnement. Nous ne retiendrons ici qu'une sélection parmi ces bouleversements. De même, nous n'évoquerons que quelques mécaniques sous-jacentes, générées par des politiques publiques, des évolutions sociétales ou des mutations technologiques, et leurs interactions.

\section{Des actifs plus nombreux, plus diplômés et plus féminins}

Deux phénomènes majeurs ont affecté l'offre de travail : une forte hausse de la population active portée par sa féminisation et l'envol du niveau d'éducation de la main-d'œuvre. Si on retient les données du Bureau international du travail (BIT) et de l'Institut national de la statistique et des études économiques (Insee), depuis 1979, la population active a progressé de près de 6 millions, s'approchant en 2019 de la barre de 30 millions de personnes. La croissance globale de la population en âge de travailler - bien plus tirée par le solde naturel que par une immigration de travail à laquelle il a été mis un terme dès le premier choc pétrolier - en explique une partie. Mais en somme, c'est bien la poursuite de l'entrée des femmes sur le marché du travail qui domine comptablement. La population active masculine a augmenté d'à peine $10 \%$ alors que la population active féminine a crû de moitié. Les femmes représentent désormais près de la moitié des actifs !

Le second phénomène est la spectaculaire montée du niveau d'éducation des actifs. Il est d'ailleurs en partie lié au premier puisque les femmes sont désormais plus diplômées que les hommes. Les données sont disponibles cette fois depuis la mise en œuvre d'une enquête Emploi modernisée en 1982. La France comptait cette année-là 2,7 millions d'actifs de niveau bac +2 ou plus, contre 11,7 millions d'actifs sans diplôme ou avec uniquement le certificat d'études primaires (CEP). En 2019, les statistiques sont inversées : autour de 11,7 millions de diplômés bac +2 et environ 3,5 millions de personnes ne disposant qu'au mieux du CEP.

Féminisation et montée du niveau d'éducation ont été, sur la période, plus rapides que ce que l'on observe en moyenne chez nos voisins. D'une part, en Allemagne ou en Italie, une vision traditionnelle de la famille a résisté, et de fait, du moins jusqu'à récemment, les politiques publiques d'accueil des jeunes enfants n'y ont pas connu la dynamique française expliquant la plus faible participation des femmes italiennes ou le taux d'emploi féminin en équivalent temps plein encore inférieur au niveau français outre-Rhin. D' autre part, la démocratisation scolaire a dû attendre en France l'arrivée au pouvoir des socialistes au début des années 1980, avec notamment la construction d'une filière de baccalauréats professionnels et la diversification de l'offre d'enseignement supérieur. Alors que la France partait d'un point bas, cet effort a 
permis d'enclencher un spectaculaire rattrapage des pays du nord de l'Europe comme de l'Amérique du Nord.

Cette démocratisation a mécaniquement réduit la participation des jeunes au marché du travail. Toutefois, l'âge des actifs n'a guère augmenté dans les deux dernières décennies du $\mathrm{Xx}^{\mathrm{e}}$ siècle car dans le même temps, la politique de retrait du marché du travail de travailleurs de plus de 55 ans, notamment peu diplômés, se poursuivait. Ce n'est que depuis le début du XXI siècle que l'effet conjugué des réformes des pensions (recul de l'âge légal, décote/surcote, cumul emploi/retraite, etc.) et de la montée en âge des baby-boomers se traduit par un vieillissement des actifs en France.

\section{Une durée réduite mais une intensification du travail}

L'élévation du niveau d'éducation de la main-d'œuvre a accompagné et probablement accéléré une reconfiguration de la demande de travail provoquée par les mutations technologiques et industrielles, et influencée par les politiques publiques. L'évolution de la durée travaillée en est une première dimension. Si on retient les données des comptes nationaux de l'Insee de 1979 à aujourd'hui, le nombre d'heures travaillées par emploi aurait diminué de plus de $15 \%$ en moyenne ; le volume total d'heures travaillées en France aurait ainsi à peine augmenté de l'ordre de $5 \%$. À la réduction de la durée collective de travail stimulée en deux temps par l'État (passage aux 39 heures hebdomadaires et à la cinquième semaine de congés payés en 1982 ; puis les deux lois Aubry, en 1998 et 2000) s'est rajoutée la montée du temps partiel, essentiellement féminin et fortement subi. L'optimisation productive dans certains secteurs explique grandement cette croissance du temps partiel. Prenons l'exemple de la grande distribution où la proportion de temps-plein a chuté depuis 1990. L'irruption du numérique (scan des produits, etc.) comme l'organisation du travail en multitâches (encaissement, remplissage des rayons libres-services, etc.) ont été une façon de répondre aux heures d'affluence des clients, mais sur des quotités significativement inférieures à un temps-plein de 35 heures, ce que l'extension des horaires et des jours d'ouverture n'a d'ailleurs guère modifié : les entreprises du secteur ont ainsi cherché à optimiser la main-d'œuvre disponible entre heures d'affluence et de creux, en privilégiant des temps partiels.

Cet exemple de densification de la durée travaillée n'est pas isolé. La réduction de la durée travaillée moyenne ne signifie pas une moindre utilisation de l'humain. La France dispose d'un outil d'observation remarquable avec ses enquêtes Conditions de travail et Surveillance médicale des expositions des salariés aux risques professionnels (Sumer) de la Direction de l'animation de la recherche, des études et des statistiques (Dares) pour cerner le phénomène d'intensification du travail qui a touché une grande part des pays de l'Organisation de coopération et de développement économiques (OCDE). La première vague remonte à 1978 ; la revue Travail et Emploi publie au début des années 1980 des travaux fondateurs méthodologiques et empiriques (par 
exemple, Volkoff, Molinié, 1982, TE $\mathrm{n}^{\circ} 11^{1}$ ) et ne cessera de valoriser un corpus de disciplines variées sur cette dimension essentielle. Dès 1996, Michel Gollac et Serge VOLKOFF (1996) constatent l'intensification et la font démarrer à la seconde moitié des années 1980. Fondamentalement, les technologies de l'information ont été mobilisées non pas pour offrir une plus grande autonomie aux travailleurs mais pour contrôler et optimiser l'usage des facteurs de production et, en premier lieu, le travail. Des pratiques organisationnelles telles les démarches qualité ou le juste-à-temps ont accentué cette dynamique au moins jusqu'à la grande récession de 2008. Elles ont participé d'un retour de gains de productivité en France comme dans de nombreux autres pays avancés (à l'exception notable de l'Italie). Mais le cumul de charge mentale et de charge physique a généré de nouvelles épidémies de pathologies professionnelles tels les troubles musculo-squelettiques, liés à ce que l'on dénomme aujourd'hui risques psychosociaux (TRAVAIL ET EMPLOI n ${ }^{\circ} 129,2012^{2}$; BODIER, WOLFF, 2018). Ces risques touchent plus particulièrement les non-cadres qui ne bénéficient pas, ou de peu d'autonomie sur l'organisation de leurs tâches. L'usure professionnelle induite interroge la capacité à long terme de ces travailleurs de demeurer en bonne santé ; son impact sur le marché du travail reste à cerner quantitativement mais pourrait être une source d'une montée particulièrement lente du taux d'emploi des plus de 55 ans en France.

\section{Des pôles tertiaires puissants}

Autre facteur d'usure des travailleurs et d' «absorption » du temps libéré par la réduction de la durée travaillée, l'allongement des trajets domicile-travail. Le « désappariement » (mismatch) spatial entre offre et demande de travail s'accentue. Les recensements montrent, depuis 1982, qu'un nombre croissant de personnes travaillent hors de leur commune de résidence et que les distances parcourues sont toujours plus importantes. Cette dynamique s'explique par une polarisation territoriale. Les emplois tendent à se concentrer dans de grands centres urbains alors que le logement devient périurbain. Cette périurbanisation est en partie subie, le prix du foncier ayant explosé dans certains centres urbains, sans que, contrairement à d'autres pays, la crise de 2008 n'apporte de correction. Tout particulièrement en Île-de-France, les moins qualifiés et les populations d'origine étrangère sont souvent relégués loin des emplois disponibles, ce qui participe d'importantes fractures spatiales (GEORGES et al., 2015).

L'agglomération des emplois dans de grands centres urbains s'explique en partie par les mutations de l'organisation des firmes et les technologies. Bien plus que stimuler le télétravail, les technologies numériques ont accru l'attractivité des centres urbains diversifiés qui proposent aux entreprises une offre variée de services (financiers, techniques, etc.). En outre, la politique française du territoire (par exemple, la décentralisation des pouvoirs) a, jusqu'à récemment, permis d'éviter que la région

1. Les références aux articles parus dans Travail et Emploi sont suivies des initiales de la revue et du numéro dans lequel le texte a été publié.

2. Notée ainsi, la référence renvoie à un numéro thématique de la revue. 
parisienne ne capture l'essentiel des bénéfices de cette dynamique (contrairement à Londres). De nombreuses capitales régionales (Lyon, Bordeaux, notamment) ont bénéficié également de politiques locales de transports qui ont permis de mettre en place des liaisons rapides avec Paris.

En miroir, des bassins d'emplois n'ont ni eu la taille critique nécessaire ni bénéficié de la volonté politique d'éviter leur déclin. Certains ont été sinistrés par la fermeture de grandes fabriques. L'industrie française n'a cependant pas disparu ; si le nombre d'heures travaillées dans la branche industrie est moitié moindre aujourd'hui qu'en 1979, une part de cette chute est liée à une externalisation dans des services aux entreprises. En outre, certains services comportent désormais des structures industrielles qui, dans les statistiques, n'apparaissent pas pour autant comme relevant de l'industrie. Par exemple, si on reste dans le domaine de la grande distribution, de nombreux hypermarchés disposent sur place, en back office, de chaînes de découpe et de conditionnement de viandes et de fromages, ou de leur boulangerie industrielle. Tous secteurs confondus et si l'on se réfère aux catégories de l'Insee, près d'un tiers des hommes sont ouvriers « qualifiés » ou « non qualifiés » aujourd'hui en France.

En revanche, la chute de l'emploi agricole est une réalité lourde qui touche de plein fouet des zones rurales. Toujours selon les comptes nationaux, le nombre d'heures travaillées dans l'agriculture a été divisé par trois en quarante ans ; leur part est tombée à $4 \%$ de la totalité des heures travaillées tous secteurs confondus malgré un temps de travail des agriculteurs et des agricultrices toujours largement supérieur à celui des autres actifs et actives.

Ce déclin est d'autant plus prononcé que l'État lui-même se désengage de ces zones rurales depuis deux décennies. Sous les deux septennats de François Mitterrand, ce désengagement n'a pas été perceptible. En effet, en répondant à la fois aux besoins éducatifs et de santé, l'emploi dans les services principalement non marchands, notamment sous un statut d'agents publics, a fortement progressé, irriguant largement le territoire. En termes d'heures travaillées totales dans l'économie française, il est passé d'un cinquième en 1979 à plus d'un quart en 1993. Toutefois, depuis maintenant vingt-cinq ans, dans un contexte de dépenses publiques contraintes, cette part ne progresse plus ; avec une population française croissante et vieillissante, les besoins augmentent et les choix politiques régionaux comme nationaux ont fait porter les moyens prioritairement sur les grandes unités urbaines. On ne parle plus de cohésion du territoire national mais - pour reprendre le titre du ministère dédié aujourd'hui - de cohésion des territoires.

\section{Plus de cadres mais une polarisation en question}

Les mutations de la demande de travail salarié comme de l'offre de travail ont également impacté massivement la répartition de l'emploi par catégories professionnelles. Si on se réfère aux catégories de l'Insee, selon les recensements, la part des cadres et professions intellectuelles a doublé depuis 1982 pour approcher les $20 \%$ au 
niveau national, et largement dépassé ce seuil dans le cœur et les banlieues proches des pôles urbains de plus de 100000 habitants. En parallèle, dans ces mêmes pôles urbains, la part des employés dits non qualifiés (CHARDON, 2001) dans l'emploi total aurait également doublé.

Ces constats sont cohérents avec des travaux concluant que la France n'échapperait pas au phénomène de polarisation professionnelle du marché du travail (RESHEFF, TOUBAL, 2019 ; TRAVAIL ET EMPLOI $\mathrm{n}^{\circ}$ 157, 2019) : les emplois les plus qualifiés seraient complémentaires des avancées technologiques récentes, les emplois de qualification intermédiaires routiniers seraient remplacés par des innovations technologiques, contrairement aux emplois non qualifiés dont la demande augmenterait notamment sous l'impulsion des consommations de services des plus qualifiés.

La polarisation demeure cependant en question. En effet, on n'observe pas en France d'augmentation des inégalités dans la distribution des salaires horaires, même si les inégalités de coûts du travail se sont accrues (voir la partie suivante). Depuis 2008, le taux d'emploi des personnes diplômées, d'un niveau bac +3 ou plus, est remarquablement stable alors qu'il a diminué pour les niveaux inférieurs et d'autant plus fortement que le niveau de diplôme est faible, suggérant plus un upgrading (une augmentation des niveaux de qualification) qu'une polarisation.

Par ailleurs, on peut mettre en débat la pertinence des statistiques publiques par catégories professionnelles (socioprofessionnelles en France) pour un suivi longitudinal de long terme. Non seulement il est difficile de faire entrer de nouvelles professions dans des catégories anciennes, mais les métiers ont connu de profondes transformations des tâches et des compétences exigées. Deux métiers en apparence proches il y a quarante ans peuvent avoir divergé tout en restant dans la même catégorie, que celle-ci soit agrégée ou détaillée. Prenons un exemple : la PCS ${ }^{3}$-2003 551a (souscatégorie de la CS 55 « employés de commerce ») regroupe toujours « gestionnaire de stock $^{4}$ » et « employé de libre-service ». Or, entre juste-à-temps et irruption du numérique, le métier de gestionnaire de stock a été bouleversé en quarante ans, subissant un fort upgrading : selon le site Orientation-pour-tous.fr sous tutelle du ministère du Travail, « un diplôme de type bac +2 tel le DUT Gestion logistique et transport permet d'accéder à ce métier. Il est également possible d'y accéder avec un bac pro Exploitation des transports ou Logistique et quelques années d'expérience ${ }^{5}$ »; le même site pour les employés de libre-service indique : «Cet emploi/métier est accessible sans diplôme ni expérience professionnelle. Un CAP/BEP en vente et distribution ou une expérience professionnelle dans ces mêmes secteurs peut en faciliter l'accès ${ }^{6}$. »

3. Profession et catégorie socioprofessionnelle.

4. S'il ne se déclare pas par ailleurs technicien, agent de maîtrise ou cadre. Un «gestionnaire des stocks » est en revanche classé dans $653 \mathrm{a}$ - donc ouvrier qualifié - ou bien en $487 \mathrm{a}$ - professions intermédiaires.

5. DUT : diplôme universitaire de technologie.

Source : https://www.orientation-pour-tous.fr/metier/gestionnaire-de-stock,13400.html, consulté le 2 août 2019.

6. CAP : certificat d'aptitude professionnelle ; BEP : brevet d'études professionnelles.

Source : https://www.orientation-pour-tous.fr/metier/mise-en-rayon-libre-service,12239.html, consulté le 2 août 2019. 
Le souci du statisticien public d'éviter des ruptures de série peut se comprendre mais peut largement biaiser le diagnostic sur l'évolution des PCS en France. Il devient même aberrant quand on voit les professeurs des écoles - catégorie A de la fonction publique de niveau master - demeurer dans les professions intermédiaires, héritage d'une époque révolue où les instituteurs étaient des fonctionnaires de catégorie B ; et ils - d'ailleurs plutôt elles - devraient demeurer dans cette case pour la future PCS 2021.

Notons que le questionnement dépasse les frontières nationales. Le BIT est rentré dans une révision de la classification ISCO (International Standard Classification of Occupations) avec probablement la remontée de certaines professions dans la hiérarchie des compétences. Des travaux de la fondation de Dublin (Eurofound, 2015) suggèrent une polarisation professionnelle lorsque les professions sont classées selon le salaire moyen par tête mais, là aussi, un upgrading lorsqu'elles sont classées par niveau moyen de diplôme observé. Une part de la polarisation salariale pourrait ainsi s'expliquer par la persistance des inégalités entre hommes et femmes, dont il sera question dans la troisième partie. Ainsi, des métiers avec une forte proportion de femmes sont en croissance, nécessitent des compétences, mais souffrent toujours d'une moindre rémunération.

\section{Chômage, précarité et permittence}

En revanche, chercheurs et statisticiens du secteur public disposent d'outils de plus en plus perfectionnés pour appréhender le chômage et la précarité professionnelle. Dès le premier numéro de Travail et Emploi (PARTRAT, 1979), chômage et politiques de l'emploi sont une préoccupation majeure, miroir de l'irruption, à la fin des années 1970, d'un chômage de masse.

Ce dernier persiste en France depuis quarante ans. Le taux de chômage BIT pour la France entière atteint les $5 \%$ en 1979. Depuis 1984, il n'est retombé en deçà de $8 \%$ que quatre années et jamais plus de deux années consécutives (en 1990, 2001-2002, 2008), avant, chaque fois, de s'envoler sous le choc d'une crise économique. La France fait ainsi figure de quasi-exception avec l'Espagne au sein des pays de l'OCDE ; même l'Italie a bénéficié d'un taux de chômage de moins de $8 \%$ pendant cinq années consécutives, tombant à $6 \%$ en 2007. La part des chômeurs de longue durée (au-delà d'un an) n'a également en France jamais reflué au-delà d'effets conjoncturels. À partir de 1979, le chômage de longue durée concerne un tiers ou plus des chômeurs (avec un pic historique en 1987 à $45 \%$ ).

Alors qu'il s'est maintenu à un niveau élevé, le chômage a vu sa composition par âge et genre évoluer. Pendant trois décennies, il s'agissait d'un phénomène plutôt féminin. Non seulement les femmes avaient un taux de chômage largement supérieur à celui des hommes, mais elles étaient aussi majoritaires au sein des chômeurs. Ce n'est que depuis dix ans, notamment du fait de l'élévation du niveau d'éducation des femmes, que les courbes se sont croisées. L'éducation est également un des facteurs 
explicatifs mécaniques de l'effondrement de la part des moins de 25 ans parmi les chômeurs : de $40 \%$ du total, les moins de 25 ans n'en représentent plus « que » $20 \%$; les guillemets s'imposent car cette proportion de $20 \%$ des moins de 25 ans ni en emploi ni en formation souligne le maintien de difficultés d'accès à l'emploi d'une part significative de la jeunesse française.

De fait, les politiques de l'emploi ciblées sur les jeunes ont été une constante des différents gouvernements (et des régions) depuis quarante ans, même si leurs paramètres ont varié : formation, embauches directes, aides et exonérations de cotisations sociales pour les entreprises, recours plus aisé aux contrats à durée déterminée (CDD). On peut largement se demander si ces politiques ont amélioré les opportunités offertes aux jeunes ou bien si elles ont participé à les enfermer dans des trajectoires de précarité ; il reste que les jeunes sont les premiers concernés par des démarrages de carrière de plus en plus hachés.

La part en légère croissance des CDD dans l'emploi salarié total depuis 1982 est trompeuse. Depuis le début du siècle, des néologismes comme le «précariat » ou la « permittence » ont été introduits pour qualifier la montée du piège que constitue une précarité permanente. La durée des contrats de travail s'est effondrée ; la médiane est aujourd'hui d'une semaine. Les remarquables enquêtes Génération du Centre d'études et de recherches sur les qualifications (Céreq) permettent de saisir l'ampleur de la dégradation et son accélération récente. Par exemple, les hommes sortis du système éducatif en 1998 étaient cinq ans après très majoritairement (59\%) en contrat à durée indéterminée (CDI) ; ceux sortis de l'école en 2010 n'étaient que $37 \%$ à être dans une telle position en 2015.

Ces nouveaux phénomènes ont poussé à étendre la notion de chômage ${ }^{7}$. La statistique publique fournit des données sur le halo du chômage, c'est-à-dire les personnes qui souhaitent travailler mais sont « classées » comme inactives selon les normes du BIT : elles ne sont pas disponibles dans les quinze jours pour travailler, ou elles ne recherchent pas activement un emploi. L'enrichissement des données de Pôle emploi avec notamment l'introduction des catégories de demandeurs d'emploi exerçant une activité réduite durant le mois de référence permet également de mieux cerner l'ampleur croissante de personnes oscillant entre emploi et non-emploi.

En outre, sous l'impulsion du Conseil national de l'information statistique (Cnis), on dispose désormais de «nouveaux indicateurs d'inégalités » qui révèlent comment la précarité professionnelle vient percuter les revenus d'activité des salariés. Ainsi, en termes réels, de 2008 à 2015, les salaires annuels nets plus les allocations chômage (donc les revenus directs et différés du travail salarié, sans inclure des prestations d'État comme la prime pour l'emploi - PPE) ont diminué pour les trois premiers déciles.

7. Pour un cadrage statistique, voir, par exemple, DE WAROQUIER DE PUEL PARLAN et al., 2018. 


\section{Une érosion du pouvoir de négociation des salariés ?}

Théoriquement, la persistance du chômage et une forte précarité ont pu participer d'un effondrement du pouvoir de négociation individuel de pans entiers du salariat. Des recherches sont en cours pour éclairer le poids d'une mécanique de monopsone en France. Là aussi, le regard a été modifié par l'amélioration du système statistique. En effet, pendant près de trente ans, analystes et politiques n'ont cessé de reprendre l'antienne que les petites et moyennes entreprises (PME) étaient les principales créatrices d'emplois en France. On sait aujourd'hui qu'il n'en était rien. Plus précisément, les grands groupes ont pratiqué une découpe en de multiples unités légales, découpe qui a longtemps brouillé le diagnostic. Le travail remarquable de profilage des groupes mené par l'Insee depuis quelques années offre un point de vue bien différent : plus du tiers de l'emploi privé est concentré dans seulement 250 entreprises en France. Cette concentration suggère qu'à l'instar des États-Unis, le marché du travail français doit présenter des situations de monopsone. Par exemple, en 2016, les groupes de plus de 5000 salariés pèsent près de $60 \%$ de l'emploi dans les grandes surfaces alimentaires et l'équipement de la maison (Rousset, 2018).

Malgré ce découpage, les capacités collectives de négociation ne sont pas nécessairement en déclin. La couverture conventionnelle de branche demeure en France supérieure à $90 \%$ alors qu'elle s'est effondrée sous l'effet des lois Hartz en Allemagne. Stimulée à partir des lois Auroux au début des années 1980 puis par une multiplicité de lois (le cas des négociations égalités professionnelles est évoqué dans la troisième partie), la négociation d'entreprise demeure dynamique, notamment dans les plus grandes structures. Par ailleurs, il n'est plus avéré que le taux de syndicalisation ait continué à s'éroder dans le dernier quart de siècle. Les enquêtes Statistiques sur les ressources et conditions de vie (SRCV) et Conditions de travail ont permis récemment de réviser nettement à la hausse les estimations de syndicalisation en France (PIGNONI, 2016). On a même des signes que la syndicalisation se serait relevée depuis 2008 dans le secteur privé (ASKENAZY, BREDA, 2019).

\section{De non-salariés à micro-entrepreneurs}

Un portrait impressionniste des grandes évolutions du marché du travail des quatre dernières décennies nécessite de s'intéresser aux non-salariés. Cette catégorie, définie par défaut, a elle aussi connu des bouleversements majeurs.

Alors que les non-salariés représentaient encore autour de $20 \%$ des personnes en emploi en 1979, leur part s'est effondrée à moins de $12 \%$ au début du siècle avant de se stabiliser, voire de légèrement augmenter depuis 2009. La France partage cette dynamique récente avec le Royaume-Uni et les Pays-Bas, dynamique qui demeure exceptionnelle au sein de l'OCDE ; ainsi, le déclin des non-salariés est marqué en part de l'emploi total comme en nombre absolu en Italie, en Espagne ou en Allemagne sur la même période. 
La baisse continue en première période de l'emploi non salarié en France est largement tirée par le recul de l'emploi agricole et de la catégorie des conjointes collaboratrices dans d'autres activités comme l'artisanat, sans être compensée par les professions libérales (médecins, avocats). La dernière décennie voit, elle, une chute du nombre des artisans classiques (métiers de l'alimentation, etc.) accélérée par la grande récession. Elle est aussi marquée par l'irruption d'emplois indépendants dans des segments comme les travaux à domicile et, plus largement, d'une nouvelle catégorie d'indépendants en emploi principal profitant de la création en 2009 du statut d'autoentrepreneurs (devenu statut de « micro-entrepreneurs »). Les données des organismes sociaux montrent que les revenus de ces derniers sont bien en deçà de ceux des indépendants classiques. L'effet de composition se voit dans les comptes nationaux : les revenus mixtes réels par heure travaillée des non-salariés auraient chuté de $20 \%$. Il s'agit d'un choc majeur pour l'emploi et le marché du travail dont les conséquences sociales - qui ont pu jouer y compris dans le mouvement d'occupations de ronds-points de l'année 2018-2019 - ont été insuffisamment explorées.

Le cumul emploi salarié et complément indépendant mériterait également plus de recherches pour saisir ses conséquences sur le marché du travail : si un tiers environ des micro-entrepreneurs ont un emploi salarié, on ne sait pas si ce cumul est nécessaire pour disposer de revenus suffisants, s'il pousse à accepter des missions ou à prendre un emploi salarié à des niveaux de rémunération faibles, etc. Le phénomène émergent du travail à la demande porté par les plateformes numériques nécessite quant à lui d'adapter l'outil statistique avant même d'essayer d'en tirer les conséquences sociétales ; le module supplémentaire de l'enquête Emploi 2017 suggérait qu'un nombre quantitativement faible de travailleurs, de l'ordre de 100 000, opéraient via ses plateformes, mais qu'en est-il aujourd'hui ? Comment faire pour capturer le micro-travail sur Internet?

En somme, les mutations du marché du travail ont été profondes et ne cessent de se renouveler. L'amélioration continue et encore nécessaire de l'appareil statistique a permis d'en suivre l'essentiel et d'éclairer ainsi le décideur public. Ce dernier a aussi bénéficié de nombreuses recherches sur les conséquences de ses actions. Ces points sont justement abordés dans la deuxième partie de cet article, consacrée aux politiques de l'emploi et à leur évaluation.

\section{Les politiques de l'emploi et leur évaluation}

Comment traiter du vaste champ des politiques de l'emploi depuis quatre décennies ? Un premier prisme s'impose presque, celui de la lutte contre le chômage : l'effet sur le chômage d'une politique est un « juge de paix » incontournable, tant du point de vue économique que politique, comme en témoigne le choix du président Hollande de faire de la baisse du chômage la condition de sa candidature à un second mandat. Bien sûr, une telle grille de lecture pousse à un constat d'échec, au moins 
partiel : quarante ans de politiques de l'emploi mobilisant un budget croissant n'ont pas endigué le chômage. Une seconde grille de lecture s'intéresse au contraire aux multiples facettes et acteurs des politiques de l'emploi, pour interroger les choix économiques et sociaux qui s'expriment ou peinent à s'exprimer à travers elles. Après tout, l'échec face au chômage tient au moins en partie à des causes extérieures au marché du travail et aux politiques de l'emploi (chocs macro-économiques, choix politiques au moment de la réunification allemande, par exemple). Et, réciproquement, les conséquences des politiques de l'emploi débordent la question du taux de chômage ou d'emploi - en touchant par exemple à l'organisation de la vie sociale ou familiale (travail le dimanche), aux inégalités sociales (revenus et transferts), etc.

Ces deux perspectives ne sont pas exclusives l'une de l'autre, ni exclusives d'autres regards. On se propose de les illustrer ici en posant trois ensembles de questions. (i) Quelles sont les principales évolutions des quatre dernières décennies - «empilement » ou émergence de «nouvelles politiques de l'emploi »? (ii) Quels sont les traits distinctifs des politiques de l'emploi en France - « exception française » ou « suivisme »? (iii) Quel rôle y a joué la recherche en économie - et quelle recherche : théorique ? appliquée ? ex ante ? ex post ? Peut-on distinguer des lignes de progrès, ou du moins d'innovation notable, dans les politiques elles-mêmes, la connaissance que nous en avons, les débats qui s'y rapportent?

\section{Empilement et déplacements des politiques de l'emploi}

«Les nouvelles politiques de l'emploi conservent la caractéristique essentielle des politiques de l'emploi des quarante dernières années, qui est le fait de procéder par empilement plutôt que par remplacement », écrit Yannick L'HorTy (2006, p. 10). La formule rend compte à la fois de la hausse continue des dépenses, et de la récurrence de nombreux dispositifs, au-delà des changements de dénomination éventuels. Ainsi, selon la Dares, les dépenses en faveur de l'emploi et du marché du travail, couvrant à la fois « les dispositifs "ciblés" sur les demandeurs d'emploi et les personnes en difficulté sur le marché du travail, et des dispositifs "généraux" destinés à réduire le coût du travail » s'élèvent en 2016 à 133 milliards d'euros, soit six points de pourcentage de produit intérieur brut (PIB), également répartis entre dépenses ciblées et générales (MONTEL, VANDERSTOCKEN, 2019) ${ }^{8}$. L'histoire des dispositifs « ciblés » est au moins aussi longue que celle de Travail et Emploi, qui a publié de nombreux articles sur les contrats aidés, les dispositifs de cessation anticipée d'activité, par exemple Certains dispositifs ont pu connaître un reflux : ainsi, les contrats aidés, en particulier ceux du secteur marchand, ont fortement décliné depuis le point haut atteint sous le

8. Sauf mention contraire, les statistiques de ce paragraphe et du suivant sont tirées de MonTEL, VANDERSTOCKEN (2019).

9. Mentionnons par exemple le numéro 16 de la revue, paru en 1983 en partie consacré au dispositif de cessation anticipée d'activité et, plus récemment, le dossier du numéro 139 de 2014 consacré à l'évaluation des politiques actives du marché du travail. 
gouvernement Jospin (900 000 bénéficiaires environ, contre 254000 aujourd'hui ; REY et al., 2018). Pour autant, sur la période 2000-2016, le constat est celui d'une relative stabilité des dépenses consacrées à ces contrats, autour de trois points de PIB, la hausse qui suit la crise de 2008 effaçant le reflux qui avait précédé (ces dépenses représentent en moyenne 2,5 points de PIB entre 2006 et 2008). En revanche, la place prise par les dispositifs " généraux » est nouvelle : elle résulte principalement de la montée continue des dépenses dues aux allègements ciblés de cotisations sociales patronales sur les bas salaires. En 2016, on compte ainsi 22 milliards d'euros d'allègements généraux de cotisations sociales sur les bas salaires, résultat d'une montée en charge quasi continue depuis les premiers allègements sous le gouvernement Balladur en 1993, et 19 milliards d'euros de crédit d'impôt pour la compétitivité et l'emploi (CICE).

Qu'en est-il de l'évolution de la structure des dépenses et, question plus subtile, du caractère innovant des dispositifs qui se sont succédé ? Au-delà de la montée des dépenses générales visant à réduire le coût du travail, on peut détecter un certain nombre d'inflexions et de ruptures. L'activation des dépenses a été l'une des évolutions revendiquées, sous l'influence en particulier de l'OCDE qui en avait fait son message évangélisateur depuis sa Stratégie pour l'emploi de 1994. Les politiques «actives » représentent ainsi, en 2016, 0,7 point de PIB, dont presque la moitié pour la formation des demandeurs d'emploi. Un autre changement notable est l'accent mis, à partir de la fin des années 1990, sur les incitations financières à l'emploi (make work pay), qui a conduit à la fois au démantèlement des dispositifs de cessation anticipée d'activité (préretraites, mais aussi dispenses de recherche d'emploi pour les chômeurs âgés), et à la mise en place de nouveaux dispositifs inspirés de l'impôt négatif des pays anglosaxons (prime pour l'emploi) ou faisant évoluer les minima sociaux de façon à limiter les effets désincitatifs sur l'offre de travail (revenu de solidarité active).

\section{Quelle place pour l'exception française?}

Parmi ces dernières tendances, l'activation des mesures ou l'accent sur l'offre de travail et les incitations financières ne distinguent guère la France des autres pays de l'OCDE, et pourraient même pousser de mauvaises langues à parler de suivisme. L'exception française, s'il y en a une, se situe davantage dans le rôle joué par les allègements de cotisations patronales sur les bas salaires et le leadership français dans le maintien d'un salaire minimum élevé, bien avant la mise en place d'un salaire minimum en Allemagne, ou les hausses récentes en diverses villes des États-Unis. Comme le montrent Antoine BozIo et ses coauteurs (2015), cette combinaison d'un salaire minimum élevé avec des allègements de cotisations concentrés autour du salaire minimum interprofessionnel de croissance (Smic) permet de revisiter la question du changement technologique biaisé et de son impact sur les inégalités. Dans ce type de contexte de politique de l'emploi, un changement technologique défavorable aux travailleurs non qualifiés n'implique pas nécessairement une explosion des inégalités 
salariales (ou la concentration du chômage sur les non-qualifiés) : s'ils sont ciblés sur les bas salaires, les allègements de cotisations patronales peuvent en effet amortir les conséquences des évolutions du coût relatif du travail payé par les entreprises. À ce titre, l'originalité des politiques françaises n'est pas d'avoir permis d'éviter un choc de demande négatif sur le travail non qualifié, qui semble largement commun aux pays de l'OCDE, mais bien d'en avoir amorti les conséquences sur les travailleurs concernés.

\section{Quel rôle de la recherche?}

Même si son rôle est difficile à objectiver, la recherche n'est pas absente des évolutions que l'on vient de décrire. Ainsi, la littérature empirique sur la perte de compétitivité en France de même que la littérature internationale sur les inégalités (puis sur la polarisation) jouent très probablement un rôle dans l'importance prise par les allègements de cotisations sur les bas salaires. Y. L'HORTY (2006) décrit les arguments théoriques des économistes en faveur d'une telle politique. Mais ces arguments n'ont pas forcément de conséquences directes sur les décisions. Ainsi, ils sont d'abord mis en avant par l'économiste Edmund Phelps aux États-Unis, lesquels n' adoptent pas cette politique. Et en France, le choix de se focaliser sur les bas salaires semble dû d'abord à un souci d'économie budgétaire, plutôt qu'aux arguments économiques portant sur la substituabilité entre travail qualifié et non qualifié. Sans doute la réalité est-elle mêlée, et la recherche en économie fournit-elle un langage et des arguments à des choix politiques et sociaux qu'elle n'influence qu'à la marge - dans le court terme du moins.

Pour dépasser ces considérations un peu générales, il est intéressant de se pencher plus précisément sur trois contributions parmi d'autres, qui ont suscité d'importants débats en France au début des années 2000 : celles de Guy LAROQUE et Bernard Salanié (2000), Bruno CréPon et Rozenn Desplatz (2001), et Olivier Blanchard et Jean Tirole (2003). Ces travaux sont de natures assez différentes. Le rapport d'O. BlanCHARD et J. TiROle s' appuie sur des recherches antérieures en partie empiriques (BLANCHARD, LANDIER, 2002). Mais le cœur de son argumentation vient de la théorie des contrats et des conclusions qu'elle permet de tirer en termes d'efficacité, de bien-être. Les auteurs y dénoncent les incitations aux effets pervers. Ils interrogent également la « rationalité » de la politique française d'indemnisation du chômage et de contrôle du licenciement, et proposent des mécanismes permettant d'améliorer l'efficacité du système selon le principe d'internalisation des externalités. Ils proposent ainsi de moduler les cotisations d'une entreprise à l' assurance chômage en fonction du nombre de salariés licenciés dans le passé, et de limiter la variabilité des indemnités prud'homales. Ils se heurtent à l'opposition d'acteurs et de chercheurs qui rappellent que ces dispositifs juridiques régissent des rapports de force inégaux entre employeurs et salariés. Les recommandations du rapport BLANCHARD-TIROLE semblent néanmoins avoir porté avec le temps puisqu'on peut penser qu'elles ont eu une influence sur la loi Travail dite loi El Khomri de 2016, en particulier sur la barémisation des indemnités prud'homales. 
Les analyses de G. LAROQUE et B. SALANIÉ (2000) allient plus directement théorie et empirie pour proposer une quantification iconoclaste de la part du « chômage volontaire » en France. La controverse suscitée par cet article est virulente. Elle est concomitante au changement d'orientation radical des politiques de l'emploi vers l'offre de travail (make work pay) dans les années 2000, évoqué plus haut. Clairement, l'ambition d'une telle décomposition est forte, peut-être trop forte au regard des données de l'enquête Emploi mobilisée dont les échantillons sont trop petits pour tirer des conclusions définitives, mais elle contribue au débat politique. On peut y lire la volonté de sortir d'une position d'experts minutieux pour mettre en perspective les politiques de l'emploi, par un diagnostic global sur le chômage.

Par contraste, la troisième contribution (CRÉPON, DESPLATZ, 2001) se veut factuelle et strictement empirique : il s'agit d'évaluer ex post l'impact des allègements de cotisations sur les bas salaires, en mobilisant des techniques micro-économétriques récentes. L'article conclut à de forts effets positifs de ces dispositifs sur l'emploi et suscite une controverse sur la plausibilité et la robustesse des résultats obtenus (selon les auteurs, les allègements de cotisations patronales auraient notamment permis des baisses sur les prix des biens relançant la demande adressée aux entreprises concernées). La politique s'installe cependant, portée successivement par les différents gouvernements de droite et gauche. Au-delà des variations d'une évaluation à l'autre, un résultat semble clair : le coût par emploi créé grâce aux allègements de cotisations est élevé.

Les conclusions que l'on peut tirer de cette rapide revue de l'interface entre recherches et politiques de l'emploi incitent à la prudence. Il ne s'agit pas d'un domaine où la recherche produit des consensus tels qu'ils s'imposent au décideur ou au débat public. Sans doute est-ce inévitable et le contraire ne serait pas souhaitable dans la mesure où les conséquences des politiques de l'emploi débordent du champ du marché du travail et engagent des conceptions de la politique et de la société.

On peut cependant souligner que l'économie du travail a connu une évolution susceptible d'être précieuse pour l'élaboration des politiques publiques et le débat public. Sans tourner le dos à la théorie, l'économie du travail est devenue au fil des ans (avec l'économie de l'éducation) un laboratoire privilégié de méthodes d'évaluation des politiques publiques, visant à une plus grande transparence et robustesse. Il est aussi possible de se mettre d'accord ex ante sur des critères et une méthode d'évaluation qui puissent être ensuite la base de constats partagés. Les expérimentations sociales contrôlées $^{10}$ qui se sont développées en France depuis le milieu des années 2000 (LABrousse, ZAMORA, 2013 et l'ensemble du numéro 135 de TRAVAIL ET EMPLOI en 2013 consacré aux expérimentations de terrain) ont ainsi produit, par exemple, des résultats qui permettent de guider l'offre de Pôle emploi vers les demandeurs d'emploi, avec, en particulier, la mise en place d'accompagnements renforcés pour ceux qui

10. Ces évaluations procèdent par l'assignation aléatoire entre un groupe « test » et un groupe « témoin ». Pour une présentation à partir d'un exemple, voir Behaghel (2012). Voir également le numéro spécial de Travail et Emploi consacré aux expérimentations de terrain (TRAVAIL ET EMPLOI $\left.\mathrm{n}^{\circ} 135,2013\right)$. 
sont le plus éloignés de la reprise d'emploi. Il a été montré que cet accompagnement pouvait être mis en œuvre de façon plus efficace par l'opérateur public que par des opérateurs privés. De façon intéressante, ce résultat et les interprétations proposées sont convergents avec l'étude sociologique qualitative de Sophie Divay (2009) et ont joué un rôle dans la stratégie suivie aujourd'hui par le service public de l'emploi ${ }^{11}$.

\section{Inégalités et discrimination}

Les transformations décrites ci-dessus du marché du travail font ressortir que la lutte contre les inégalités d'accès à l'emploi et de déroulement de carrière entre les individus tient aujourd'hui une place centrale dans les politiques publiques. De ce fait, les décideurs ont besoin de mieux connaître les mécanismes sous-jacents et les différences de traitement selon les caractéristiques personnelles. Parallèlement, d'un point de vue conceptuel, l'une des évolutions majeures depuis le milieu des années 1980 des recherches en économie du travail est que l'homo economicus, concept central du raisonnement micro-économique, s'est vu doté d'un sexe, d'une origine, d'une famille, de voisins, de normes sociales, etc. Ces attributs, dont on le débarrassait pour construire des modèles simples d'offre de travail ou de consommation, sont devenus essentiels pour comprendre ce qui se passe sur les marchés et dans l'entreprise. En se donnant un ancrage historique, social, psychologique, le champ de la recherche en micro-économie du travail s'élargit dans un dialogue fructueux avec les autres sciences humaines, démarche qui a d'ailleurs toujours été présente dans la revue Travail et Emploi. Cette évolution de la discipline va au-delà du simple ajout de variables dans des représentations économiques qui resteraient fondamentalement les mêmes ; la diversité humaine, les multiples facettes des interactions sociales sont devenues au cœur des recherches en économie.

Nous présenterons ici quelques résultats dans ce champ de recherche, en nous concentrant sur deux dimensions pour lesquelles les travaux sont nombreux et ont beaucoup évolué dans les dernières décennies, à savoir le genre et l'origine. Ces deux dimensions sont centrales à la fois parce que la lutte contre les discriminations est au cœur des débats publics et parce que, malgré une sensibilité croissante à ce sujet et un renforcement des législations antidiscriminatoires, les inégalités perdurent. Dans le cas de la situation des femmes par rapport aux hommes, plus de participation sur le marché du travail et plus d'éducation n'ont pas suffi à éliminer les inégalités ; dans celui de l'origine, au surchômage des immigrés s'est ajouté le chômage massif des descendants d'immigrés d'origine non européenne, pourtant nés et éduqués en France.

11. Combinés avec d'autres résultats d'expérience (BEHAGHEL et al., 2013). 


\section{Le genre : d'un thème mineur à un point de passage obligé des politiques du travail et de l'emploi}

Les recherches autour de la place des femmes sur le marché du travail sont aujourd'hui abondantes et on ne pourrait pas imaginer une conférence académique sur le « Travail » sans au moins une à deux sessions sur le genre. La place croissante de ce thème dans les revues spécialisées, dont Travail et Emploi ${ }^{12}$, et la multiplicité des sujets abordés sont significatives de cette évolution. Dans ce texte, nous présenterons brièvement les grandes évolutions de la participation des femmes au marché du travail, ses conséquences sur les inégalités salariales et la mesure de la discrimination, les répercussions sur les cadres législatifs et réglementaires et sur ce qui se passe lorsqu'on ajoute l'enfant dans ces réflexions.

Si les différences de travail et de rémunération selon le sexe n'ont jamais été complètement ignorées par les économistes, ce thème a longtemps été considéré comme mineur. La sous-représentation des femmes dans le travail marchand était vue comme une conséquence de la division des tâches au sein des ménages. Les femmes constituaient une force d'appoint, fluctuant au gré de la conjoncture. Claudia GoLDIN (1998), professeure en économie à Harvard, ancienne présidente de l'American Economic Association et référence majeure sur ce domaine, en expliquant comment elle a conduit ses recherches sur l'histoire de l'emploi des femmes aux États-Unis, se remémore qu'elle a soudain réalisé que les statistiques des années 1930 aux États-Unis avaient un point aveugle : les femmes mariées. Alors que l'évolution des normes sociales et des emplois avait conduit depuis la fin de la Première Guerre mondiale à considérer comme une position acceptable pour une épouse d'avoir un emploi salarié, la crise économique eut pour conséquence de rendre prioritaire l'emploi masculin et de rejeter les femmes à la maison. Les entreprises aux États-Unis mais aussi dans d'autres pays (Irlande, Royaume-Uni, Japon par exemple) pouvaient alors appliquer une discrimination légale à l'encontre des femmes mariées qualifiées (institutrices principalement, ou employées qualifiées) en refusant de les embaucher ou en les licenciant après le mariage. Après la Seconde Guerre mondiale, le besoin de main-d'œuvre qualifiée fit tomber en désuétude cette disposition.

Mais la rupture la plus importante dans la question de l'analyse des inégalités entre les femmes et les hommes est celle appelée « la révolution tranquille » (GoLDIN, 2006) quand la diffusion de la pilule contraceptive changea la donne pour les nouvelles générations. La maîtrise du calendrier de la fécondité permit aux jeunes filles de se

\footnotetext{
12. Dès 1985, Travail et Emploi publiait un article sur l'égalité salariale entre les femmes et les hommes (BUGHIN, PAYEN, 1985, TE $\mathrm{n}^{\circ} 23$ ). Les auteurs concluaient que les différences constatées provenaient essentiellement de la ségrégation sexuée des emplois (secteurs, qualifications). La loi « à travail égal, salaire égal » était donc globalement respectée. Il est frappant de constater qu'à l'époque, l'article ne se posait pas la question de l'accès des femmes aux différents niveaux hiérarchiques, pas plus qu'il n'envisageait que la ségrégation professionnelle entre dans le champ de l'évaluation de la discrimination salariale. Depuis, de nombreux articles sont parus sur le sujet dans Travail et Emploi, ainsi que des numéros dédiés à des thèmes peu explorés, comme « Genre et travail indépendant 》 (TRAVAIL ET EMPLOI, $\left.\mathrm{n}^{\circ} 150,2017\right)$.
} 
projeter dans des études longues et de se construire une identité professionnelle, facilitée par le développement des services dans l'économie. À grands traits, les femmes arrivées sur le marché du travail à la fin des années 1990 sont de moins en moins différentes des hommes en termes de nombre d'années d'études et de comportement d'activité. Comme cela a été souligné au début de ces contributions, dans les dernières générations, les femmes sont même davantage diplômées que les hommes.

Ces transformations affectent les inégalités et leurs mesures. Depuis OAXACA (1973) et BLINDER (1973), il est devenu standard de décomposer l'écart de rémunération moyen entre les hommes et les femmes en une part expliquée par les différences de caractéristiques productives entre les deux groupes (éducation, expérience et, selon les spécifications adoptées, les caractéristiques de l'emploi occupé), et une part qui reste inexpliquée correspondant à un rendement supérieur de ces caractéristiques chez les hommes par rapport aux femmes. Cette part inexpliquée est souvent interprétée comme un indicateur de l'ampleur de la discrimination salariale. Il ressort des travaux empiriques une tendance nette, commune à l'ensemble des pays de l'OCDE : l'écart brut de rémunération entre les femmes et les hommes ne décroît plus, ou très lentement, et la part expliquée diminue ; la quasi-totalité de l'écart est inexpliquée et ne repose donc plus sur les différences observées de capital humain (éducation, expérience).

Le regard s'est alors déplacé des différences des moyennes vers celles des distributions des revenus, avec l'observation de la raréfaction des femmes dans les emplois les mieux rémunérés. L'expression « plafond de verre » va devenir un thème central, résumant le fait que les carrières des femmes stagnent parfois sans que l'on puisse expliquer exactement pourquoi. Au plafond de verre va s'ajouter le «plancher collant », désignant les cas où les femmes restent plus longtemps que leurs homologues masculins dans le bas des échelons de leur emploi. Les recherches autour de ces constats font éclater les frontières traditionnelles de la micro-économie en mettant en avant les traits psychologiques, selon lesquels les femmes auraient une plus forte aversion au risque, hésiteraient à s'engager dans des compétitions, ou à négocier des salaires plus avantageux ou une promotion ; ces recherches tiennent également compte de l'identité sexuée dont dépendent les choix de spécialisation scolaire et de professions, de la résistance des hommes à la féminisation de « leurs » professions, du degré de féminisation de la profession et bien sûr des arrangements domestiques au détriment des femmes, que nous évoquons plus bas.

Afin de briser de manière institutionnelle le plafond de verre au sommet de la hiérarchie des emplois, des quotas ont été imposés dans les conseils d'administration et dans la haute fonction publique, avec une sanction financière en cas de non-observation. Dans le cas de la France et du secteur privé, la loi dite Copé-Zimmermann a instauré en 2011 l'obligation d'un quota de $40 \%$ de femmes dans ces conseils des grandes sociétés cotées françaises. La conséquence a été immédiate, puisque le taux de femmes dans ces conseils est monté très vite, mais s'est accompagné d'un plafond de verre interne, les femmes n'y occupant pas les postes les plus prestigieux et rémunérateurs (REBÉRIOUX, RoudAut, 2016). 
Au-delà des conseils d'administration, l'inégalité des salaires a fait l'objet d'une succession de lois pour contraindre les entreprises à respecter l'égalité, chaque texte précisant ce qui lui semblait central. En France, les principaux jalons sont la loi sur l'égalité salariale de 1972, la loi Roudy de 1983 avec l'introduction du rapport de situation comparée pour servir de base chiffrée dans les discussions avec les partenaires sociaux, la loi Génisson de 2001 qui mettait l'accent sur les carrières, ou la loi VallaudBelkacem, de 2014 sur l'égalité réelle, avec une attention portée à la conciliation entre vie familiale et professionnelle. Ces lois avaient en commun d'imposer de nouvelles règles dans l'entreprise pour atteindre l'égalité professionnelle, mais sans fixer d'objectifs. Le décret du 8 janvier 2019 qui met en place l'index « Pénicaud Schiappa » de l'égalité femmes-hommes opère une rupture dans ces approches puisqu'il oblige les entreprises à publier un indicateur standardisé $e^{13}$, avec une obligation de résultat en trois ans, assortie d'une menace d'une sanction financière égale à $1 \%$ de la masse des rémunérations si l'entreprise n'atteint pas un nombre de points défini.

Mais le facteur central de la persistance de l'inégalité salariale entre femmes et hommes, bien connu depuis longtemps, a pris une importance nouvelle avec l'atténuation, voire la disparition des causes antérieures (différences de participation, de niveau d'éducation) : ce sont désormais les maternités et leurs conséquences qui expliquent la quasi-totalité des inégalités salariales. Pendant de l'inertie de l'écart salarial, le partage des tâches domestiques au sein des familles s'est peu modifié au fil du temps. Les femmes prennent toujours la part du lion (CHAMPAGNE et al., 2015) et ce, d'autant plus que l'arrivée d'un enfant a des répercussions sur le travail, les horaires, le temps partiel, et peut se traduire par un retrait temporaire d'activité.

Une vaste littérature s'est développée pour évaluer les pénalités salariales liées à la maternité, en comparant femmes avec et sans enfants, le manque à gagner résultant massivement du retrait temporaire du marché du travail ou de la diminution des heures travaillées (KLEVEN et al., 2018). Le problème dans cette perspective est de distinguer ce qui relève de l'offre de travail (arbitrage personnel ou familial) de ce qui provient de la demande (les employeurs freinent la carrière des mères). Quels que soient les méthodes statistiques choisies ou les pays étudiés, les travaux économiques mettent en évidence des conséquences négatives à long terme sur les salaires (et donc sur les retraites) de plus longues interruptions d'activité ${ }^{14}$. Notons que des politiques publiques non ciblées sur les mères peuvent aussi avoir un effet sur leur participation dès lors qu'elles touchent les rythmes scolaires et sociaux. Ainsi, la réforme (interrompue) de l'école le mercredi s'est immédiatement traduite par un accroissement des mercredis travaillés des mères (DUCHINI, VAN EFFENTERRE, 2018), ce qui confirme que la charge de la conciliation repose toujours principalement sur elles et que beaucoup souhaiteraient en être libérées.

13. Sur les débats autour de cet indice, CoRON et al., 2019.

14. Pour la France, voir PiketTy (2005), et LEQuiEN. (2012). 
Cette dernière réflexion nous amène directement du côté des pères. Les quelques recherches sur les effets de la paternité dans le travail convergent pour dire que celle-ci n'affecte pas grand-chose, ni la participation, ni les heures travaillées, et peut-être même que cela aurait un effet positif sur les rémunérations.

En revanche, on constate depuis quelques années une poussée des politiques en faveur de longs congés paternités, surtout dans les pays nordiques, avec souvent des quotas réservés aux pères dans le total du congé parental. Sur ce sujet, la France est à la traîne avec seulement onze jours calendaires à prendre pour les pères ; certaines entreprises prennent d'ailleurs l'initiative d'allonger ces congés rémunérés ou de favoriser l'expression des besoins des pères pour prendre soin des enfants. Cela suffira-t-il pour rééquilibrer et partager la charge mentale?

\section{Des origines plus visibles?}

Le deuxième thème abordé dans cette partie est toujours en rapport avec l'identité sociale de l'agent économique, mais nous nous intéressons ici au fait qu'il soit un(e) Français(e) d'origine hexagonale. La question des discriminations ethniques est depuis très longtemps étudiée par les économistes américains à travers deux mécanismes différents. La discrimination basée sur les préférences des agents (BECKER, 1957) part du principe que les individus (employeurs, collègues, clients, etc.) peuvent avoir une aversion particulière à l'encontre d'un groupe démographique. La discrimination statistique (PHELPS, 1972 ; ARROW, 1973) considère que tous les agents sont rationnels et qu'ils n'ont pas de réticence particulière envers un groupe démographique. Néanmoins, la productivité des individus qui postulent à des offres d'emploi est difficile à anticiper si bien que les recruteurs se servent des informations disponibles pour faire la meilleure prévision possible de celle-ci. Si la productivité supposée du groupe ethnique minoritaire est plus faible en moyenne ou plus dispersée, les recruteurs utilisent l'ethnie du candidat pour pénaliser tous les curriculum vitae (CV) venant de ce groupe, ce qui engendre une diminution des recrutements de ce même groupe.

En France, la thématique des discriminations à l'encontre des minorités ethniques a surtout émergé à la suite de la marche pour l'égalité et contre le racisme de 1983. Auparavant, la question des inégalités portait principalement sur l'intégration des immigrés sur le marché du travail français. L'état de la connaissance sur ces discriminations s'est sensiblement amélioré au cours des dix dernières années. En effet, l'existence d'une discrimination ethnique ${ }^{15}$ sur le marché du travail est désormais largement documentée par de nombreux travaux empiriques utilisant principalement deux méthodes complémentaires : d'un côté, l'analyse des inégalités associées à l'origine ethnique et non expliquées par les caractéristiques sociodémographiques (à l'aide d'enquêtes statistiques) et de l'autre, les expérimentations aléatoires (testings).

15. On désigne ici comme « ethnie » un groupe qui a en commun une ascendance, une histoire, une culture, une langue ou un dialecte. 
Cependant, si ce phénomène est largement confirmé par différentes études, les sources en restent encore largement inconnues. Or connaître ces dernières est primordial pour mettre en place des politiques publiques efficaces.

Pour mesurer et lutter contre les discriminations à l'encontre des minorités ethniques, il faut pouvoir identifier les individus discriminés. La loi Informatique et libertés de janvier 1978 interdit les statistiques ethniques, mais des dérogations sont permises pour que les chercheurs puissent mesurer ces discriminations et comprendre leurs mécanismes ${ }^{16}$. Ainsi, depuis le début des années 2000, plusieurs enquêtes ont été créées ou enrichies pour permettre d'étudier la situation des immigrés et descendants d'immigrés sur le marché du travail français : enquête Formation et qualification professionnelle 2003 (Insee), enquêtes Emploi (Insee) et enquêtes Trajectoires et origines 2008-2009 et bientôt 2019-2020 (Institut national d'études démographiques [Ined], Insee). Dans ces enquêtes, la mention de la nationalité de naissance du ou des parents immigrés permet d'approcher l'ethnicité des individus.

Ainsi, plusieurs auteurs ont utilisé ces enquêtes pour décrire la situation des immigrés et de leurs descendants sur le marché du travail français. D’un point de vue général, ces études empiriques montrent une plus grande difficulté d'accès à l'emploi pour ceux d'origine « extra-européenne » (maghrébine et africaine) que pour les Hexagonaux. Ce constat est d'autant plus intéressant que ce moindre accès ne s'explique qu'en partie par leurs caractéristiques individuelles propres (âge, localisation et niveau de diplôme) $)^{17}$.

À l'inverse de l'approche indirecte, la méthode expérimentale permet de révéler les comportements discriminatoires par la réaction des agents à une situation construite par les chercheurs. Ainsi, plusieurs études de testing visant spécifiquement à identifier des comportements de recrutement discriminatoires ont récemment été menées ${ }^{18}$.

Un test de discrimination par paire consiste à proposer sur une même offre d'emploi deux candidatures statistiquement équivalentes du point de vue de la formation, de l'expérience professionnelle et des caractéristiques sociodémographiques (sexe, âge, diplôme, nationalité et lieu de résidence). La seule différence entre les deux candidatures réside dans l'origine ethnique, avec des noms et prénoms à consonance « hexagonale » pour l'une et à consonance « non hexagonale » pour l'autre. La mesure de la discrimination nécessite de confronter les réponses faites à l'une et à l'autre candidature pour chaque test. On considère comme un résultat positif une invitation de l'employeur à participer à un entretien d'embauche. En comparant le taux moyen

\footnotetext{
16. La collecte et l'utilisation de statistiques ethniques sont un sujet très débattu en France à l'inverse des pays anglosaxons. Ceux qui s'y opposent y voient un moyen de catégoriser statistiquement la population française, fragilisant la culture universaliste.

17. Cependant, la part non expliquée par ces caractéristiques observables ne peut être attribuée entièrement à de la discrimination, car toute caractéristique inexpliquée corrélée avec l'origine ethnique et non entièrement corrélée avec les variables explicatives peut affecter cette partie inexpliquée. Il est donc nécessaire d'interpréter avec précaution les résultats utilisant cette approche d'analyse des résidus.

18. L'article de Marianne Bertrand et Sendhil Mullainathan (2004) qui examine distinctement l'ampleur de la discrimination raciale à l'embauche pour les hommes et pour les femmes fait référence dans le domaine.
} 
de réponses positives entre le groupe minoritaire et le groupe majoritaire, on peut ainsi tester l'existence d'une discrimination ethnique à un entretien d'embauche (qui est la première étape d'un accès à l'emploi).

Plusieurs expérimentations effectuées au cours des dernières années ont mis en évidence, en France comme dans de nombreux pays, l'existence de comportements discriminatoires relativement répandus chez les employeurs lors du recrutement ${ }^{19}$, de sorte que nous disposons aujourd'hui d'un ensemble d'éléments empiriques attestant l'existence de discriminations à l'encontre des individus issus des minorités ethniques (origine suggérée par le patronyme) sur le marché du travail français (par exemple, CÉDIEY et al., 2008 ; DUGUET et al., 2010). Plusieurs testings révèlent ainsi que les travailleurs d'origine supposée « maghrébine » sont particulièrement touchés par le phénomène ${ }^{20}$. En fonction des propriétés de l'expérimentation (métiers, période, caractéristiques des candidats, etc.), à caractéristiques similaires, les candidats d'origine supposée « hexagonale » ont jusqu'à trois fois plus de chances de recevoir un retour positif des employeurs que ceux d'origine supposée « maghrébine ».

À l'heure actuelle, les analyses utilisant ces deux approches permettent difficilement de savoir quels mécanismes sont précisément à l'œuvre. Si ces discriminations sont aujourd'hui avérées notamment dans les études qui utilisent des méthodes de testing, il y a encore trop peu d'études qui portent sur des mesures qui permettent de les réduire efficacement. Certains travaux empiriques récents étudient la question des possibilités d'action pour lutter contre ce phénomène. Luc BEHAGHEL et ses coauteurs (2015) ont expérimenté la mise en place d'une politique d'anonymisation des CV (absence d'informations liées à l'ethnie) mais leurs résultats n'ont toutefois pas permis de conclure sur l'efficacité d'une telle mesure. Florent FREMIGACCI et ses coauteurs (2015) ont évalué l'impact de la labélisation du mérite, en faisant apparaître sur le CV l'obtention de la distinction «meilleur apprenti de France », mais il semble que cela soit peu efficace pour réduire les discriminations puisque les candidats d'origine « hexagonale » bénéficient davantage de cette distinction que les candidats minoritaires. Enfin, A. EDO et N. JACQUEMET (2013) évaluent l'effet du signalement d'une bonne maîtrise du français sur les $\mathrm{CV}$. Leurs résultats montrent qu'un tel signal permet de réduire marginalement les écarts observés.

Face à l'existence avérée des discriminations, plusieurs pays ont cherché à favoriser les initiatives émanant directement du secteur économique pour lutter contre les discriminations à l'embauche. Aux États-Unis, dans les années 1980, on a ainsi assisté à l'essor du diversity management et, plus récemment en France, à la signature d'une charte de la diversité suivie par la promotion d'un label diversité que les entreprises peuvent obtenir si elles affichent leur engagement pour le respect de l'égalité de traitement et de la non-discrimination.

19. Voir par exemple Bertrand, Duflo (2017), et Neumark (2018) pour des revues de littérature.

20. Voir notamment l'étude de Clémence BERSON (2013, TE $\left.\mathrm{n}^{\circ} 135\right)$ sur les emplois d'hôtes de caisse dans la grande distribution, ainsi que celle d'Anthony ÉDO et Nicolas JACQUEMET (2013). 
Aux États-Unis, des formations « diversité » qui cherchent à agir directement sur le processus de recrutement en formant les recruteurs pour réduire leurs biais afin de diminuer leur propension à discriminer ${ }^{21}$ ont vu le jour. Ces formations ont aussi connu une forte augmentation en France ces dernières années. Une obligation de formation des recruteurs à la non-discrimination pour les entreprises de plus de 300 salariés a d'ailleurs été inscrite dans la loi Égalité et citoyenneté du 27 janvier 2017. Il serait très utile que les chercheurs mènent des expérimentations en collaboration avec les entreprises pour évaluer l'efficacité sur la discrimination à l'embauche des formations à la diversité telles qu'elles se pratiquent aujourd'hui en France. De manière générale, une coopération plus étroite entre ces deux parties pour réfléchir à la mise en place de politiques publiques efficaces bénéficierait à tous.

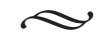

Que concluraient aujourd'hui nos deux Persans ? Ils pourraient, indubitablement, revendiquer certaines permanences, et retrouver en France aujourd'hui une partie des idées portées par l'École de Chicago hier. Le capital humain se recycle en investissement social, et le revenu minimum et l'impôt négatif font leur retour en cour. La discrimination basée sur les préférences et sa persistance à l'équilibre n'ont rien perdu de leur actualité. Certaines mutations du travail étaient, elles, plus difficiles à prévoir. Auraient-ils vu venir les défis posés par les données massives (big data) pour la connaissance des comportements individuels et la statistique publique ? Les enjeux du numérique et ceux liés à la transition écologique pour l'emploi de demain ? Auraient-ils imaginé les revendications sociales portées par le mouvement des Gilets jaunes ? Quant à la discipline elle-même, nos Persans auraient certainement été surpris de voir la place qu'y ont prise les recherches appliquées, souvent économétriques. C'est quand même beau, Paris.

\section{BIBLIOGRAPHIE}

Arrow K. J. (1973), “The Theory of Discrimination”, in Ashenfelter O., Rees A. (eds.), Discrimination in Labor Markets, Princeton (N. J.), Princeton University Press, 1973, pp. 3-33.

Askenazy P., Breda T. (2019), “Electoral Democracy at Work”, Working Paper, n 2019-42, Paris School of Economics.

BeCKer G. S. (1957), The Economics of Discrimination, Chicago, Chicago University Press.

Behaghel L. (2012), «Un exemple d'expérimentation sociale contrôlée : le cas du CV anonyme », Informations sociales, ${ }^{\circ} 174$, pp. 62-65.

21. En effet, certains travaux ont mis en évidence que les stéréotypes des recruteurs sont responsables d'une part non négligeable de leurs comportements discriminatoires (cf. la revue de littérature de M. BERTRAND et E. DuFLo [2017]). 
Behaghel L., Crépon B., Gurgand M., Kamionka T., Lequien L., Rathelot R., Zamora P. (2013), « L'accompagnement personnalisé des demandeurs d'emploi », Revue française d'économie, vol. XXVIII, $\mathrm{n}^{\circ} 1$, pp. 123-158.

Behaghel L., Crépon B., Le Barbanchon T. (2015), "Unintended Effects of Anonymous Resumes", American Economic Journal: Applied Economics, vol. 7, n 3 3, pp. 1-27. https:// doi.org/10.1257/app.20140185.

BERSON C. (2013), «Testing : les difficultés de l'interprétation de la discrimination à l'embauche », Travail et Emploi, n ${ }^{\circ} 135$, pp. 27-40.

Bertrand M., Duflo E. (2017), "Field Experiments on Discrimination", in Banerjee A. V., Duflo E. (dir.), Handbook of Field Experiments, vol. 1, Amsterdam, North Holland, pp. 309-393.

Bertrand M., Mullainathan S. (2004), “Are Emily and Greg More Employable Than Lakisha and Jamal? A Field Experiment on Labor Market Discrimination", American Economic Review, vol. 94, nº 4, pp. 991-1013. https://doi.org/10.1257/0002828042002561.

Blanchard O., Landier A. (2002), “The Perverse Effects of Partial Labour Market Reform: Fixed-Term Contracts in France", The Economic Journal, vol. 112, n 480, pp. F214-F244. https://doi.org/10.1111/1468-0297.00047.

Blanchard O., Tirole J. (2003), Protection de l'emploi et procédures de licenciement, Paris, La Documentation française, coll. «Les rapports du Conseil d'analyse économique ».

Blinder A. S. (1973), "Wage Discrimination: Reduced Form and Structural Estimates", The Journal of Human Resources, vol. 8, n 4, pp. 436-455.

Bodier M., WolfF L. (2018), Les Facteurs psychosociaux de risque au travail : d'après le rapport d'expertise sur le suivi statistique des risques psychosociaux au travail présidé par Michel Gollac, Toulouse, Octarès.

Bozio A., Breda T., Guillot M. (2015), “Taxes and Technological Determinants of Wage Inequalities: France 1976-2010”, Working Paper, n 2015-05, Paris School of Economics.

Bughin E., PAYen J.-F. (1985), «Les disparités des salaires des hommes et des femmes », Travail et Emploi, $\mathrm{n}^{\circ} 23$, pp. 49-53.

CÉDIEY É., Foroni F., GARNER H. (2008), « Discriminations à l'embauche fondées sur l'origine à l'encontre de jeunes français(es) peu qualifié(es). Une enquête nationale par tests de discrimination ou testing », Premières informations, premières synthèses, n ${ }^{\circ}$ 06.3, Dares.

Champagne C., Pailhé A., Solaz A. (2015), « Le temps domestique et parental des hommes et des femmes : quels facteurs d'évolutions en 25 ans ? », Économie et statistique, $\mathrm{n}^{\circ}$ 478-479480, pp. 209-242.

Chardon O. (2001), « Les transformations de l'emploi non qualifié depuis vingt ans », Insee première, $\mathrm{n}^{\mathrm{o}} 796$.

Coron C., Boussard-Verrecchia E., Berthou K., Meurs D. (2019), « Controverse. Quelle efficacité attendre des indicateurs pour mesurer les écarts de rémunération entre les femmes et les hommes ? », Revue de droit du travail, $\mathrm{n}^{\circ} 3$, pp 147-160. 
Crépon B., Desplatz R. (2001), « Une nouvelle évaluation des effets des allégements de charges sociales sur les bas salaires », Économie et statistique, ${ }^{\circ}$ 348, pp. 3-24.

Duchini E., VAn Effenterre C. (2018), Do Women Want to Work More or More Regularly? Evidence from a Natural Experiment, version d'octobre 2018, Working paper, Paris School of Economics.

Edo A., JACQUEMET N. (2013), « Discrimination à l'embauche selon l'origine et le genre : défiance indifférenciée ou ciblée sur certains groupes ? ", Économie et statistique, n ${ }^{\circ} 464$ 465-466, pp. 155-172.

Duguet E., Leandri N., L'Horty Y., Petit P. (2010), “Are Young French Jobseekers of Ethnic Immigrant Origin Discriminated against? A Controlled Experiment in the Paris Area", Annals of Economics and Statistics, n ${ }^{\circ}$ 99-100, pp. 187-215.

Eurofound (2015), Upgrading or Polarisation? Long-Term and Global Shifts in the Employment Structure: European Jobs Monitor 2015, Luxembourg, Publications Office of the European Union.

Fremigacci F., Parquet L. (DU), Petit P., Pierné G. (2015), « Le mérite : un rempart contre les discriminations ? », Revue française d'économie, vol. 30, nº 1, pp. 155-182.

Georges N., L'Horty Y., SARI F. (2015), « Comment réduire la fracture spatiale ? Une application en Île-de-France », Économie \& prévision, n ${ }^{0}$ 206-207, pp. 17-37.

Goldin C. (1998), "The Economist as Detective", in Szenberg M., Passion and Craft: Economists at Work, Ann Arbor, University of Michigan Press, pp. 98-112.

Goldin C. (2006), “The Quiet Revolution That Transformed Women's Employment, Education, and Family", American Economic Review, vol. 96, n 2, pp. 1-21. https://doi. org/10.1257/000282806777212350.

Gollac M., Volkoff S. (1996), « Citius, altius, fortius. L'intensification du travail », Actes de la recherche en sciences sociales, $\mathrm{n}^{\circ} 114$, pp. 54-67.

Kleven H., Landais C., Søgaard J. E. (2018), “Children and Gender Inequality: Evidence from Denmark", NBER Working Paper, n 24219, National Bureau of Economic Research.

LABrousse A., ZAMORA P. (2013), «Expérimentations de terrain et politiques publiques du travail et de l'emploi. Apports récents et mises en perspective. Introduction », Travail et Emploi, $\mathrm{n}^{\mathrm{o}} 135$, pp. 5-13.

LARoQue G., SAlaniÉ B. (2000), « Une décomposition du non-emploi en France », Économie et statistique, $\mathrm{n}^{\circ} 331, \mathrm{pp} .47-66$.

LEQuiEn L. (2012), “The Impact of Parental Leave Duration on Later Wages", Annals of Economics and Statistics, $\mathrm{n}^{\circ} 107-108$, pp. 267-285.

L'Horty Y. (2006), Les Nouvelles Politiques de l'emploi, Paris, La Découverte.

Montel O., Vanderstocken A. (2019), « Les dépenses en faveur de l'emploi et du marché du travail en 2016. Une nouvelle forte hausse des dépenses générales », Dares résultats, nº 007.

Neumark D. (2018), "Experimental Research on Labor Market Discrimination", Journal of Economic Literature, vol. 56, n 3, pp. 799-866. https://doi.org/10.1257/jel.20161309. 
OAXACA R. (1973), "Male-Female Wage Differentials in Urban Labor Markets", International Economic Review, vol. 14, n 3, pp. 693-709.

PARTRAT M. (1979), «Évolution récente et caractéristiques actuelles du chômage des jeunes », Travail et Emploi, $\mathrm{n}^{\circ} 1$, pp. 51-63.

Phelps E. S. (1972), “The Statistical Theory of Racism and Sexism”, American Economic Review, vol. 62, n ${ }^{\circ}$, pp. 659-661.

Pignoni M.-T. (2016), « La syndicalisation en France. Des salariés deux fois plus syndiqués dans la fonction publique », Dares analyses, $\mathrm{n}^{\circ} 025$.

PIKETTY T. (2005), « Impact de l'allocation parentale d'éducation sur l'activité féminine et la fécondité en France, 1983-2002 », in Lefèvre C., Filhon A. (dir.), Histoires de familles, histoires familiales. Les résultats de l'enquête Famille de 1999, Paris, Ined, pp. 79-109.

Rebérioux A., Roudaut G. (2016), “Gender Quota and Inequalities inside the Boardroom”, Document de travail (Docweb), $\mathrm{n}^{\circ}$ 1603, Cepremap.

Resheff A., Toubal T. (2019), La Polarisation de l'emploi en France (1994-2018). Ce qui s'est aggravé depuis la crise de 2008, Paris, Rue d'Ulm.

Rey M., Bellit S., Guillon V., Firquet S., Mourlot L. (2018), « Les dispositifs spécifiques d'emploi aidé et de formation au $3^{\mathrm{e}}$ trimestre 2018. Poursuite de la baisse du nombre de personnes en contrat aidé », Dares indicateurs, $\mathrm{n}^{\circ} 062$.

Rousset A. (2018), « Portrait de groupes dans le commerce. Un poids élevé et une forte spécialisation », Insee première, $\mathrm{n}^{\circ} 1724$.

TRAVAIL ET EMPLOI (2012), dossier « Les risques psychosociaux au travail : d'une "question de société" à des questions scientifiques », nº 129.

TRAVAIL ET EMPLOI (2013), dossier « Expérimentations de terrain et politiques publiques du travail et de l'emploi. Apports récents et mises en perspective », $\mathrm{n}^{\circ} 135$.

TRAVAIL ET EMPLOI (2014), dossier «Évaluation des politiques actives du marché du travail », $\mathrm{n}^{\mathrm{o}} 139$.

TRAVAIL ET EMPLOI (2019), dossier "Polarization(s) in Labour Markets", n’ 157.

Volkoff S., MoliniÉ A.-F. (1982), «Quantifier les conditions de travail ? (L'exemple de l'enquête nationale d'octobre 1978) », Travail et Emploi, n 11, pp. 63-70.

Waroquier de Puel Parlan S. (de), Dixte C., Guillaneuf J., Minni C., Rebiere S., Rey M. (2018), «Emploi, chômage, population active en 2017 : nouvelle accélération de l'emploi salarié privé, et amplification de la baisse du chômage », Dares analyses, $\mathrm{n}^{\circ} 31$. 
